\title{
Determinación de la Efectividad de la Remediación de Suelos Accidentalmente Contaminados con Ácido Sulfúrico mediante Métodos Geofísicos
}

\author{
Ignacio D. Coria ${ }^{(1)}$ y Matías de la Vega ${ }^{(2)}$ \\ (1) Facultad de Ciencias Empresariales, Universidad Abierta Interamericana, Av. Pellegrini 1957, \\ S2000BUG Rosario-Argentina (e-mail: dcoria@sinectis.com.ar) \\ (2) Departamento de Geofísica Aplicada, Facultad de Ciencias Exactas y Naturales, \\ Universidad de Buenos Aires - CONICET, Int. Güiraldes 2160 - Ciudad Universitaria C1428EGA, \\ Buenos Aires-Argentina (e-mail: matias@df.uba.edu.ar)
}

Recibido Mar. 04, 2010; Aceptado Abr. 19, 2010; Versión Final recibida May. 28, 2010

\begin{abstract}
Resumen
Este trabajo muestra que el diagnóstico de suelos contaminados con sustancias peligrosas después de la remediación puede realizarse con técnicas no invasivas, en menos tiempo, con menor costo y evitando los riesgos de contaminación cruzada de muestras del método invasivo. Los métodos no invasivos utilizados para este caso particular de una contaminación accidental con ácido sulfúrico concentrado fueron el de inducción electromagnética y el método geoeléctrico. De acuerdo con los resultados obtenidos, se concluye que la utilización complementaria de ambos métodos permite realizar un diagnóstico correcto de la efectividad de la remediación.
\end{abstract}

Palabras clave: inducción electromagnética, método geoeléctrico, contaminación de suelo, efectividad de remediación, diagnóstico invasivo

\section{Determination of the Remediation Effectiveness of Soils Accidentally Contaminated by Sulphuric Acid by Geophysical Methods}

\begin{abstract}
This work demonstrates that the diagnosis of soils that have been contaminated with dangerous substances after remediation can be done with non invasive techniques, with cost and time reduction, and avoiding the sample cross contamination risks of invasive methods. The noninvasive methods used in this particular case of accidental contamination with concentrated sulphuric acid were electromagnetic induction and geoelectric. According to the results, it is concluded that the complementary use of both methods allows making a correct diagnosis of the remediation effectiveness.
\end{abstract}

Keywords: electromagnetic induction, geoelectric method, soil contamination, remediation effectiveness, invasive diagnosis 


\section{INTRODUCCIÓN}

Los métodos de diagnóstico de suelos contaminados por sustancias peligrosas pueden clasificarse en invasivos (tradicionales), practicando perforaciones en suelo contaminado y extrayendo muestras a distintas profundidades para análisis químicos, y no invasivos (geofísicos): geoeléctrico e inducción electromagnética (EMI), entre otros. En general, en los métodos geofísicos con una fuente (externa o natural; eléctrica, magnética, elástica, etc.) se excita el subsuelo y a partir de la respuesta obtenida se infiere la distribución de alguna propiedad del subsuelo (resistividad, constante dieléctrica, elasticidad, etc. Ver por ejemplo: Telford et al., 1990). Para obtener una imagen de la distribución de alguna de estas propiedades es necesario resolver lo que se conoce como problema inverso: conocida la fuente de excitación y la respuesta que produce, hay que caracterizar las propiedades del medio. Este problema en sí mismo no tiene una solución única. Además, hay que tener en cuenta que los materiales tienen distintas respuestas frente a los distintos tipos de excitación y por lo tanto ciertos métodos pueden dar muy buena respuesta mientras que otros pueden fracasar, ya sea local o regionalmente. Por eso, para mejorar la resolución, es necesario aplicar distintas técnicas que contribuyan a disminuir la ambigüedad de las soluciones (Coria et al., 2009). De hecho se han encontrado evidencias de una mejor resolución cuando se combinan métodos alternativos y distintas configuraciones (Lascano et al., 2003).

Dentro de los métodos geofísicos como el geoeléctrico y el de inducción electromagnética, la caracterización de plumas contaminantes se hace a partir de los cambios en la resistividad del subsuelo debido a la presencia del contaminante (Stemberg et al. 1990). Con la información recabada se puede estimar el estado del suelo y la profundidad de las napas, y se extrapola una posible "pluma contaminante". La caracterización de plumas contaminantes producidas por derrames de diversas sustancias se realiza generalmente mediante métodos invasivos, seguidos de la aplicación de modelos matemáticos; sin embargo, los métodos geofísicos son menos costosos y tienen las ventajas de reducir los tiempos de diagnóstico y evitar la contaminación cruzada de muestras, lo cual ocurre con frecuencia en el muestreo invasivo. La caracterización de zonas contaminadas por métodos geofísicos está ampliamente documentada en la bibliografía (Walraevens et al., 1997; Benson et al., 1997; Atekwana et al., 2000; Osella et al., 2002; Shevnin et al., 2006; Coria et al., 2009). Con estos métodos, se puede determinar la extensión de una pluma contaminante y en caso de que ésta llegue a la napa de agua, detectar la presencia y extensión del sobrenadante (de la Vega et al., 2003). La combinación con técnicas petrofísicas permite determinar las concentraciones de contaminantes (Shevnin et al., 2005). También, se han utilizado métodos geofísicos en la etapa post remediación, como en el control de la efectividad de la remediación en el caso de un sitio contaminado con gasolina (Coria et al., 2009). En el presente trabajo se estudia la factibilidad de utilizar los métodos geofísicos de inducción electromagnética y geoeléctrica en la evaluación de la efectividad de un proceso de remediación de suelo contaminado por un derrame de ácido sulfúrico.

\section{MATERIALES Y METODOS}

En febrero de 2006, un accidente automovilístico produjo el vuelco de un camión que transportaba 22.000 litros de ácido sulfúrico al 98 \% p/p, en Ruta 60, Km. 870, en las cercanías de Quilino (provincia de Córdoba, Argentina).

El método de diagnóstico post-derrame empleado fue el de análisis químico mediante perforación de pozos. La Tabla 1 muestra los análisis de acidez del suelo antes de la remediación. Las muestras se tomaron en el Pozo 1, ubicado en el centro del derrame (véase Fig. 1).

Tabla 1: Resultados de los análisis de acidez del suelo antes de la remediación

\begin{tabular}{|c|l|l|l|}
\hline Horizonte & Profundidad & $\mathrm{pH}$ & Características \\
\hline $\mathrm{A}$ & $0-20 \mathrm{~cm}$ & 1,5 & Textura franco limosa con presencia de materia orgánica \\
\hline $\mathrm{B}$ & $20-40 \mathrm{~cm}$ & $4.0-6,2$ & Textura franco limosa \\
\hline $\mathrm{C}$ & $40-60 \mathrm{~cm}$ & 7,2 & Textura franco limosa \\
\hline
\end{tabular}




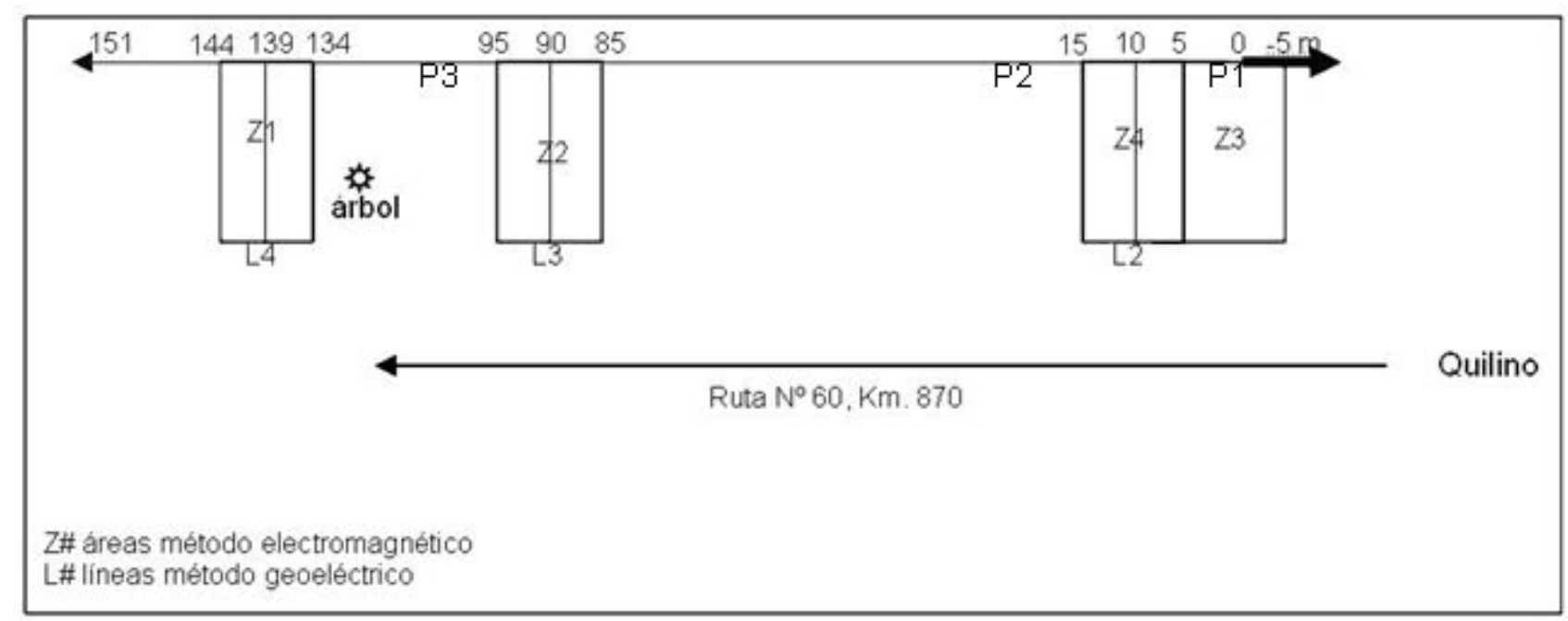

Fig. 1: Croquis del sitio analizado por métodos no invasivos

Mediante estos datos se delimitó el área afectada y el grado de dispersión del contaminante tanto en profundidad como en forma superficial. El derrame de ácido sulfúrico llevó el pH del suelo de 7 a 1,5 en zonas impactadas y los sulfatos a 1,62 \% p/p. La superficie total impactada fue de aproximadamente $1.000 \mathrm{~m}^{2}$ y la profundidad máxima, de $0,5 \mathrm{~m}$. Se realizó un censo de pozos para la determinación de la posición y movilidad del cuerpo de agua freática y analizar la vulnerabilidad de contaminación de la misma. Se realizaron calicatas con toma de muestras de suelos en los diferentes horizontes diagnósticos identificados para determinar los valores de $\mathrm{pH}$ que dieran indicios confiables del grado de contaminación del suelo y sus variaciones tanto en sentido horizontal como vertical. Para ello se realizaron tomas en diferentes sectores del área contaminada. Las muestras fueron analizadas en el Laboratorio de Suelos y Aguas de la Agencia Córdoba Ambiente, provincia de Córdoba, Argentina. Se trata de una contaminación del tipo puntual con un mecanismo de infiltración difusa o generalizada que afectó solamente a un área de extensión limitada, constituida por la presencia del contaminante ácido con escasa a nula capacidad de infiltración por debajo de los 0,40-0,50 m debido a la presencia de un horizonte con una textura limo arcillosa que lo hace muy poco permeable.

Se ve que la contaminación es superficial y que llega hasta 0,5 metros de profundidad en toda la zona impactada. En base a esto, el método de remediación escogido fue el de remediación in situ con neutralización y estabilización química de la acidez de los suelos mediante la utilización de un álcali de gran potencia. Se realizaron dos etapas: 1) Shock alcalínico en la mancha de mayor concentración ácida y 2) Neutralización y estabilización química del área afectada. La primera etapa consistió en la rápida neutralización del horizonte $A$, que presenta mayor contaminación, llevando su pH de 1,5 a 4 mediante la adición de $\mathrm{Ca}(\mathrm{OH})_{2}$ en una proporción o concentración mayor al 5\%. La cantidad total de hidróxido de calcio empleada fue de $32 \mathrm{Tn}$, a partir de cálculo estequiométrico. En la segunda etapa se continuó con la neutralización de la totalidad de la zona afectada mediante incorporación de $\mathrm{Ca}(\mathrm{OH})_{2}$ en una proporción del $4 \%$. En ambas etapas se procedió por escarificación y mezcla de la totalidad del suelo con el álcali y posterior riego.

\section{RESULTADOS OBTENIDOS EN LA EVALUACION DE LA REMEDIACION}

El estudio post remediación, llevado a cabo para evaluar la efectividad de la misma, se realizó tanto con la técnica invasiva habitual como con los métodos geoeléctrico e inducción electromagnética. El monitoreo de evaluación tradicional, realizado en forma simultánea con el desarrollo de las tareas de remediación corresponden a los pozos de P1 a P5, ubicados (véase Fig. 1): P1 en el centro del derrame, P2 a 40 m, P3 a 115 m, P4 a 200 m y P5 a 280 m del centro del derrame en la dirección sur-norte, con una profundidad de 0 a 0,40 m. Para las mediciones con métodos de diagnóstico no invasivos, se utilizaron los métodos geoeléctrico y de inducción electromagnética. En la Fig. 1 se muestra la zonificación del área y las mediciones realizadas; Z1 
hasta Z4 corresponden a las áreas estudiadas con el método de inducción electromagnética, y con L2 hasta L4 se indican las líneas prospectadas con el método geoeléctrico.

El área prospectada con inducción electromagnética Z4 corresponde a la zona del derrame y Z2 a la zona de escurrimiento, mientras que Z1 se halla fuera de la zona afectada. Z3 es contigua pero externa a la zona afectada. L2, L3 y L4 son las líneas prospectadas con el método geoeléctrico. El punto 0 corresponde a la zona de derrame del ácido sulfúrico concentrado (Z3) y dada la pendiente superficial hacia el oeste se realizaron mediciones electromagnéticas hasta $\mathrm{Z1}$, ubicada entre 134 y $144 \mathrm{~m}$ de distancia, y mediciones con método geoeléctrico hasta L4 a $139 \mathrm{~m}$ del punto 0 .

Con el método geoeléctrico se realizaron en total tres perfiles (ver Fig. 1) en configuración dipolodipolo realizando en cada caso dos series de mediciones, una con separación entre electrodos de $1 \mathrm{~m}$ y otra de $0,5 \mathrm{~m}(\mathrm{a}=1$ y $0.5 \mathrm{~m})$. En todos los casos la separación máxima entre los electrodos de corriente y potencial fue de seis veces estas distancias ( $n=6$, ver por ejemplo Telford, 1990). Se utilizó esta configuración para, combinando en cada perfil las dos series de mediciones, obtener al invertir una buena resolución lateral y a su vez poder llegar a una profundidad de sondeo del orden de los $3 \mathrm{~m}$. Para la inversión de los datos se utilizó el programa Res2DInv (Loke y Barker, 1996) combinando los datos correspondientes a las aberturas de 0,5 y $1 \mathrm{~m}$ para tener buena resolución en las capas más superficiales y a la vez mayor poder de penetración.

En la Fig. 2 se muestran los resultados obtenidos de la inversión de los datos tomados. En esta figura, la dirección vertical indica profundidad y la horizontal distancia creciente hacia la ruta. En la escala de grises se muestran los valores de resistividad del subsuelo. De arriba hacia abajo, las líneas son: L2, L3 y L4.

Los estudios de inducción electromagnética se realizaron con un equipo Geophex GEM-2. Este equipo puede emitir hasta quince frecuencias en el rango entre 330 y $47970 \mathrm{~Hz}$. En la práctica, se trabajó con seis frecuencias para aprovechar mejor la potencia emitida y a la vez disponer de datos correspondientes a distintas profundidades. Las seis frecuencias seleccionadas fueron mayores a los $5000 \mathrm{~Hz}$ ya que las profundidades de interés eran menores a los $3 \mathrm{~m}$. Se tomaron datos en las áreas indicadas a lo largo de las líneas con una separación de $1 \mathrm{~m}$ entre línea y línea. En la Fig. 3 se muestran los resultados obtenidos en las cuatro zonas, interpolando los datos obtenidos en las líneas de cada zona para mostrar un barrido en superficie.
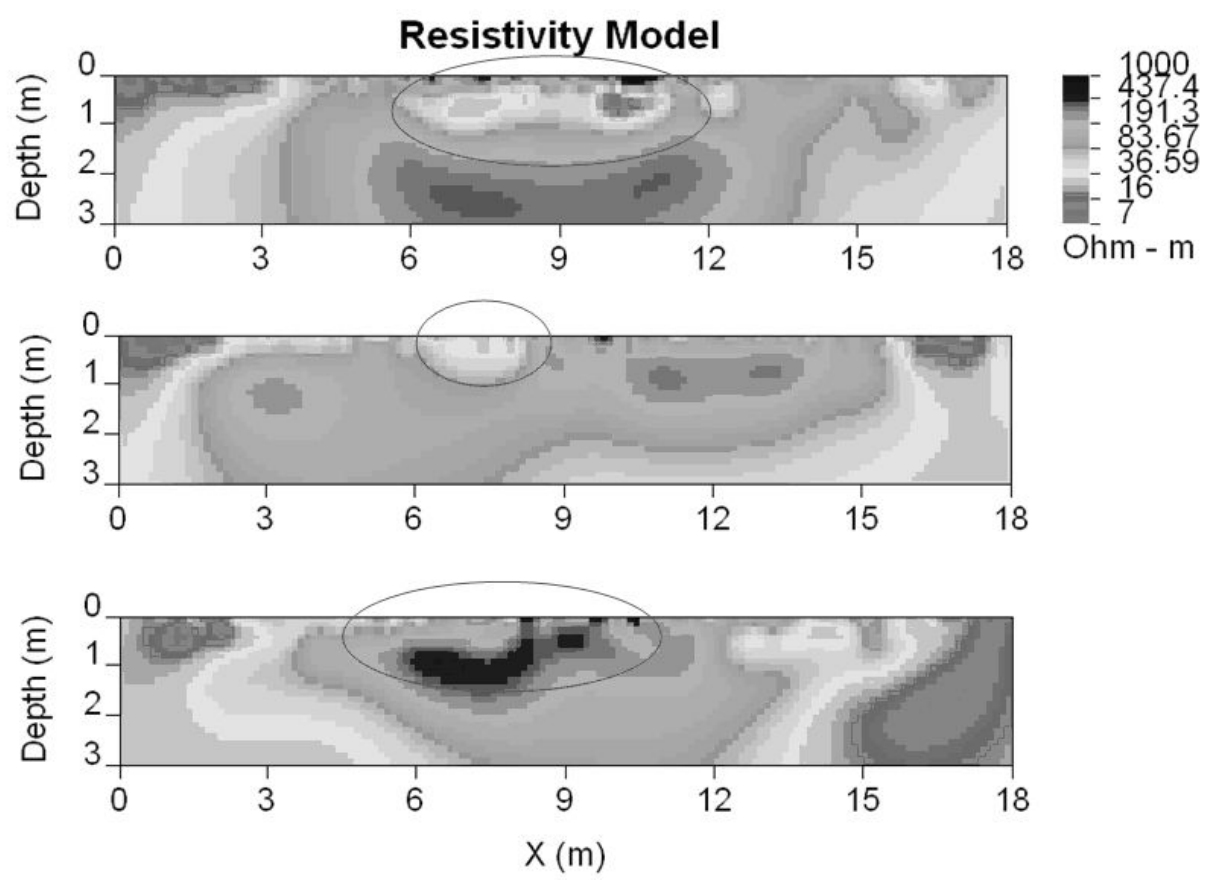

Fig. 2: Resultados obtenidos por el método geoeléctrico 


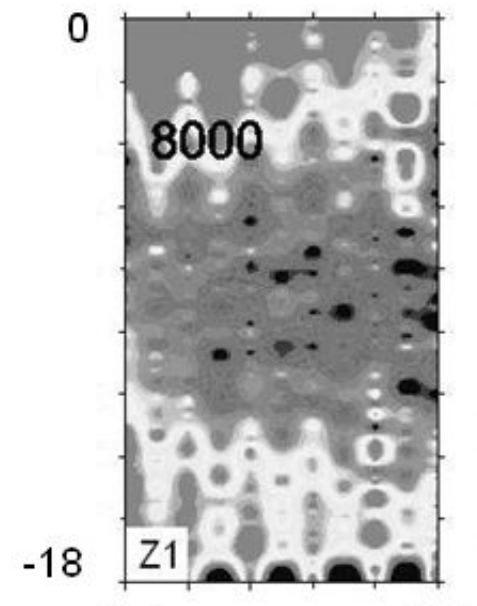

$-144$

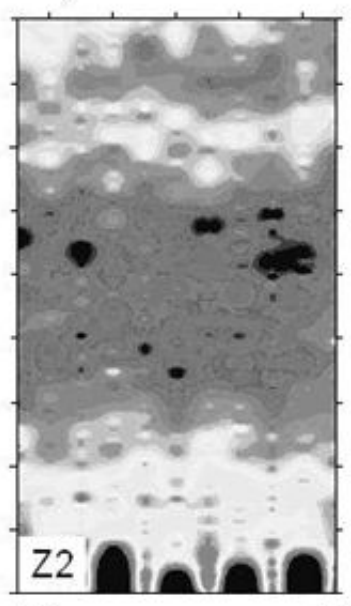

$-86-14$

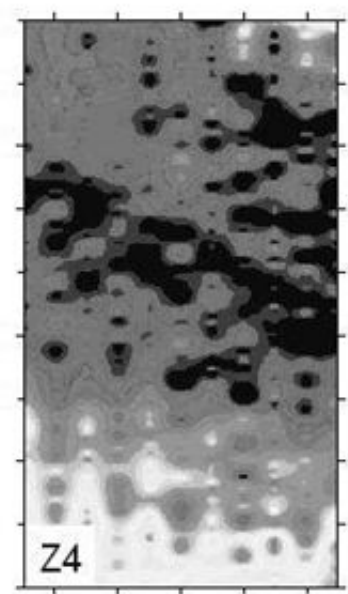

$-6$

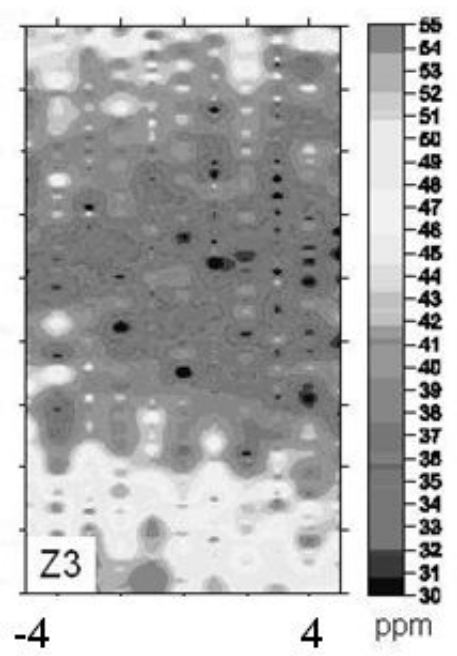

Fig. 3: Resultados obtenidos por el método de inducción electromagnética (EMI)

La dirección vertical indica distancia hacia la ruta y la dirección horizontal indica la distancia paralela a la ruta. La escala de grises indica el campo diferencial detectado por la segunda espira en ppm. Aquí se muestran los resultados correspondientes a una sola frecuencia $(8000 \mathrm{~Hz})$. Para frecuencias menores, correspondiente a capas más profundas, los gráficos son parecidos entre sí, indicando un subsuelo homogéneo a más profundidad. Para frecuencias mayores, que corresponden a lo más superficial, los datos son similares a los aquí mostrados.

\section{DISCUSIÓN}

Los valores de $\mathrm{pH}$ en las muestras tomadas en los mencionados pozos muestran el incremento de la alcalinidad producido por el tratamiento fisicoquímico de neutralización realizado, y que los valores de $\mathrm{pH}$ alcanzados son similares a los de un suelo no contaminado.

Tabla 2: Análisis de acidez del suelo después de la remediación (Agencia Córdoba Ambiente, provincia de Córdoba, Argentina)

\begin{tabular}{|c|c|c|c|c|c|}
\hline & P1 & P2 & P3 & P4 & P5 \\
\hline pH en $\mathrm{H}_{2} \mathrm{O}$ & 12,0 & 11,4 & 6,9 & 8,6 & 7,8 \\
\hline
\end{tabular}

En las mediciones con método geoeléctrico se observa que en la línea $L 2$, que corresponde a la zona más contaminada, hay un cambio de resistividad entre 0 y $1 \mathrm{~m}$ de profundidad. La anomalía más significativa presenta una resistividad de alrededor de $10 \mathrm{ohm} . \mathrm{m}$. En la línea L3, que corresponde a la zona de escurrimiento del contaminante, se observa una anomalía de similar resistividad ( 10 ohm.m) a menor profundidad, y de menor ancho que en la línea L2. En L4, fuera de la zona contaminada, no se observan anomalías de este tipo. La aplicación de este método, por lo tanto, demuestra que la alta resistividad del suelo evidencia que éste no contiene remanente de un electrolito como el ácido sulfúrico.

En el caso de inducción electromagnética, aunque los resultados son cualitativos, también se observan tres zonas diferenciadas. Una zona oscura amplia en Z4 que se corresponde con la zona donde fue el derrame y se realizó la remediación, que se repite en Z2 aunque en forma más estrecha, coherente con la zona de escurrimiento. Las otras dos zonas presentan características similares y se corresponden con zonas no afectadas. La aplicación de este método, por lo tanto, indica qué parte del suelo fue impactada, realizando un barrido superficial. Que la remediación ha sido eficiente tanto en el pozo testigo como en el resto de la zona remediada puede comprobarse por el análisis químico del pozo testigo y por el valor de resistividad homogéneo en toda la zona remediada. Se comprueba que la delimitación de la zona contaminada ha sido adecuada. A la vez, se comprueba que la contaminación era superficial. 


\section{CONCLUSIONES}

Se pueden obtener las siguientes conclusiones principales: i) Los métodos no invasivos permiten realizar monitoreos post remediación para verificar la eficiencia del tratamiento empleado; ii) El diagnóstico de suelos contaminados por ácido sulfúrico y posteriormente remediados puede realizarse con ventajas técnicas y económicas por métodos no invasivos, como EMI y geoeléctrico, respecto al tradicional método invasivo por perforación de pozos; iii) Los métodos EMI y geoeléctrico pueden utilizarse, además, en forma complementaria para lograr mayor certeza respecto a los datos obtenidos por método invasivo; iv) El método de remediación empleado (fisicoquímico por neutralización) fue el correcto para este caso particular; y v) La eficiencia de la remediación en este caso particular se ha comprobado tanto por un método invasivo (análisis químico sobre muestras de suelo extraídas por perforación) como por dos métodos no invasivos (geoeléctrico y EMI).

\section{AGRADECIMIENTOS}

A la Lic. María Victoria Bongiovanni, por su labor en la inversión de datos en los métodos geoeléctrico y EMI. A La Segunda, Compañía Limitada de Seguros Generales, por el apoyo económico para la cobertura de gastos de movilidad y estadía en Quilino.

\section{REFERENCIAS}

Atekwana, E.A., W.A. Sauck y D.D. Werkema, Investigations of geoelectrical signaturas at a hydrocarbon contaminated site, Journal of Applied Geophysics, 44, 167-180 (2000).

Benson, A.K., K.L. Payne y M.A. Stubben, Mapping ground-water contamination using dc resistivity and VLF geophysical methods, Geophysics, 62, 80-86 (1997).

Coria, I. D., V. Bongiovanni, N. Bonomo, M. de la Vega y M.T. Garea, Hydrocarbon Contaminated Soil: Geophysical-Chemical Methods for Designing Remediation Strategies, Near Surface Geophysics, 7 (3), 227-236 (2009).

de la Vega, M., A. Osella y E. Lascano, Joint inversion of Wenner and dipole-dipole data to study a gasoline contaminated zone, Journal of Applied Geophysics, 54, 97-109 (2003).

Lascano, E., A. Osella, M. de la Vega, S. Buscaglia, X. Senatore y J.L. Lanata, Geophysical Prospection at Floridablanca Archaeological Site, San Julian Bay, Argentina, Archaeological Prospection, 10, 175-192 (2003).

Loke, M.H. y R.D. Barrer, Res2Dinv. Coded based on rapad least-squares inversion of apparent resistivity pseudosections by a quasi-Newton method, Geophysical Prospecting, 44, 131-152 (1996).

Osella, A., M. de la Vega y M. Lascano, Characterization of a contaminated pluma due to a hydrocarbon spill using geoelectrical methods, Journal of Environmental and Engineering Geophysics, 7 (2), 78-87 (2002).

Shevnin, V., O. Delgado-Rodríguez, A. Mousatov, D. Flores Hernández, H. Zegarra Martínez y A. Ryjov, Estimation of soil petrophysical parameters from resistivity data: Application to oil-contaminated site characterization, Geofísica Internacional, 45 (3), 179-193 (2006).

Shevnin, V., O. Delgado-Rodríguez, L. Fernández-Linares, H. Zegarra Martínez, A. Mousatov y A. Ryjov, $G$ characterization of an oil-contaminated site in Tabasco, México. Geofísica Internacional, 44 (3), 251-263 (2005).

Telford, W.M., L.P. Geldart y R.E. Sheriff, Applied Geophysics, Cambridge University Press, Cambridge (1990).

Walraevens, K., E. Beeuwsaert y W. De Breuk, Geophysical methods for prospecting industrial pollution; a case history, European Journal of Environmental and Engineering Geophysics, 2, 95-109 (1997). 